\title{
DETERMINING COOPERATION IN MULTIAGENT SYSTEMS WITH CULTURAL TRAITS
}

\author{
Stefan Heinrich, Stefan Wermter \\ Department of Informatics, Hamburg University, Hamburg, Germany \\ heinrich@informatik.uni-hamburg.de,wermter@informatik.uni-hamburg.de \\ Markus Eberling \\ Department of Computer Science, University of Paderborn, Paderborn, Germany \\ markus.eberling@upb.de
}

Keywords: Adaptation, Altruism, Cooperation, Cultural traits, Multiagent Systems.

\begin{abstract}
Achieving cooperation among autonomous and rational agents is still a major challenge. In the past, altruistic cooperation was generally explained through genetic kinship relations. However, the theory of 'cultural kin' is an approach that tries to explain altruism through cultural relatedness. To promote cooperation among autonomous and rational agents, this work transfers the idea of cultural characteristics, which benefits social behaviour, to multiagent systems (MAS). Accordingly, agents are characterised by cultural traits, which they can imitate from their neighbours and are supposed to solve tasks, for which they need the cooperation of other agents in most cases. The interaction of cooperation and cultural trait propagation will be investigated in a theoretical analysis and in an empirical simulation in a particular developed framework. As a novelty, schemata will be analysed that are beyond the well-studied one-to-one interaction.
\end{abstract}

\section{INTRODUCTION}

During the last years the interest in multiagent systems has increased noticeably (Shoham and LeytonBrown, 2008). The idea of solving problems by distributing them among autonomous agents was taken up continually in theoretical and practical contexts. Moreover, problem areas have emerged, in which autonomous entities are supposed to optimally exploit limited resources through the use of cooperation (for example bandwidth or computation capacity of peer-to-peer networks). An issue, which is still not satisfactorily dealt with, is how cooperation can be achieved among those autonomous and rational agents, if there is the appeal of cheating.

The contribution of this work is an analytical analysis of a modelled multiagent system, which was proposed similarly in (Hales, 2001; Klemm et al., 2005) and (Eberling, 2009). The agents of such a MAS carry several cultural traits but have only limited local knowledge of their neighbours' traits. Additionally, our agents hold weightings of theirs traits, which are completely invisible to others. Every agent randomly and continuously receives jobs, most of which he can only solve with the help of cooperating and altruistic partners. Similar to the prisoner's dilemma, the global benefit for all agents strongly increases if cooperation - preferably of a reciprocal nature - takes place. Agents adapt to their best neighbours through imitation, which implies that particular trait values will be propagated more intensively than others. However we will show that this propagation of cultural traits is limited by the agents' fundamental disposition to cooperate. The model's dynamics and the model itself have been discussed for several decades but the new analytical approaches provide a better understanding of these models and cooperation in MAS in general.

In the field of biology and other disciplines explanations for cooperation and reciprocal altruism were initially found exclusively in the kinship relations: supporting related organisms - even at one's own expenses - will serve the own genes and thus indirectly yourself (Axelrod and Hamilton, 1981; Trivers, 1971). Cultural norms, which seem to be responsible for altruism in the absence of genetic relatedness, and their propagation in societies, was a topic of intensive research during the last decades (Allison, 1992; Axelrod, 1986; Axelrod, 1997; Binmore, 1998; Boyd and Richerson, 1985; Delgado, 2002). In conjunction with norms, ideas and behavioural patterns were un- 
derstood as cultural traits, which represent a cultural relatedness (Dawkins, 1976). Under the metaphor of cultural evolution (Heylighen, 1992), cooperation on the basis of cultural traits as well as their propagation were further analysed. Transferred to the context of artificial societies or in particular to multiagent systems, they were increasingly labelled as 'features', 'memes' or 'tags'.

With his Swap Shop Framework, Hales (Hales, 2001) analysed the cooperation in artificial societies: agents shared resources that were essential for survival on the basis of cultural relatedness (memetic kin). For this reason each agent carried three cultural traits memes including an altruistic trait and was able to learn traits from his neighbours. In Hales empirical analysis, setups with cultural evolution as well as with genetic evolution lead to an identical dominance of single groups with a specific trait-setting. Nevertheless, they occurred much faster within the cultural evolution which provides some evidence of the impact of cultural traits. Furthermore, he found out that the distribution of resources through cultural evolution was more efficient and lead to a more social behaviour.

On the basis of Axelrod's Cultural Diffusion Model (Axelrod, 1997) Klemm et al. evaluated under which terms the propagation of cultural traits converges to stable conditions and what those might look like (Klemm et al., 2005). Their agents were connected through a one-dimensional lattice and had various features, which they infrequently adapted to a feature of one of their neighbours. The adaptation was only depending on a initial existence of a common feature. Depending on the amount of possible traits per feature and the amount of the features themselves, the simulation arrived at an equilibrium: for an initially high diversity of the features in a polarised condition (several small homogeneous groups had evolved) and for an initially low diversity in a globalised condition (consistently homogeneous agents). For each setting Klemm et al. determined a threshold, under which a polarisation and over which a globalization occurred.

From the literature, we can adopt valuable approaches, which follow the idea empirically or analytically in small cases. With our work, we want to go a step further and contribute analytical results for more than a one-to-one interaction scheme.

In the next section we will formally present our model. In section 3 we will present a theoretical analysis of the model and subsequently demonstrate some substantiating experiments in section 4 . In the last section we will draw a conclusion and give an outlook to future work.

\section{THE MODEL}

In this section we will outline our formal model. The major aim of our concept is a multiagent system, which follows the ideas of the literature (see above) but still remains computable. In particular, we use discrete ranges of sizes which are large enough but finite for all parameters.

\subsection{Formal MAS Description}

\section{Definition 1 (World)}

The world which contains the agents $\mathcal{A}$ is a twodimensional square lattice of a finite size $m \times n$ with the following parameters:

- $|\mathcal{A}|=m \cdot n:$ the number of agents is defined by the size of the lattice; every cell represents an agent

- Moore neighbourhood relation (see Figure 1)

- $z \in \mathbb{N}$ : range dimension of cultural trait values

- the world is a torus in order to avoid boundary problems, therefore cell $(m+1, n+1)=$ cell $(1,1)$

The dynamics of the world is divided in discrete time slots (t), so-called rounds. At the outset the world is initialised with the parameters and is static in the size of the lattice, and thereby in its size of population. All agents are distinctly defined by their position in the world. The neighbours are the set of agents who are known and available for interaction. The range dimension of cultural trait values defines the range $\{1, \ldots, z\}$, in which the values of cultural traits can be located.

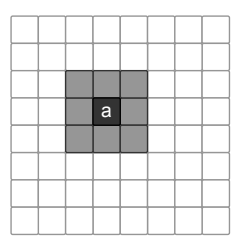

Figure 1: Moore neighbourhood.

Definition 2 (Agent)

An agent $a \in \mathcal{A}$ is defined as $a=\langle\mathcal{N}, f, g, q, s\rangle$ with:

- $\mathcal{N} \subseteq \mathcal{A}$ : neighbours of a, according to the neighbourhood relation

- $f=(u, v, w), \quad u, v, w \in\{1, \ldots, z\}$ : cultural traits

- $\left.g=\left(g_{u}, g_{v}, g_{w}\right), \quad g_{u}, g_{v}, g_{w} \in\right] 0,1\left[, \quad g_{u}+g_{v}+\right.$ $g_{w}=1:$ trait weightings

- $q \in\{0, \ldots, z-1\}$ : altruism threshold

- $s \in \mathbb{Z}$ : score, counting rewards and costs 
The position of the agents results from the initialisation of the world. At the beginning the score is at 0 and the agents' cultural traits are randomly selected from the uniform distribution $U(1, z)$. To determine the weightings, the range $[0,1]$ is divided at the intersections $\zeta_{1}$ and $\zeta_{2}$ to receive three weightings according to the length of the intervals $\left[0, \zeta_{1}\right],\left[\zeta_{1}, \zeta_{2}\right]$, and $\left[\zeta_{2}, 1\right]$. Those weightings are randomly assigned to the weightings $g_{u}, g_{v}$, and $g_{w}$. The altruism threshold defines the largest value of cultural difference, up to which a cooperation is agreed on, and in this approach is a free variable (details below).

\section{Definition 3 (Jobs)}

The set $\mathcal{I}$ is made of jobs $j=\langle a, e\rangle$ with:

- $a \in \mathcal{A}$ : agent to whom the job was assigned

- $e \in\left\{1, \ldots,\left|\mathcal{N}_{a}\right|+1\right\}$ : effort (amount of agents needed) to solve the job

For solving an assigned job, the agent receives $2 \cdot e$ points. Every agent involved in solving this job, has to pay costs of one point.

\subsection{Behavioural Heuristics}

Taken from the knowledge about human social behaviour, all agents follow two fundamental behavioural heuristics, which psychologists and sociologists assume to be most likely (Allison, 1992; Cialdini, 1994; Noble and Franks, 2004):

1. Be good to your close relatives (agents expect a higher reciprocity from similar agents)

2. Imitate those who are successful (these agents seem to have traits, which make them more successful)

Implementing heuristic 1 means that every agent $b \in \mathcal{N}_{a}$ cooperates with agent $a$, if the values of the cultural traits - with respect to the trait weightings - do not vary widely from the values of the cultural traits of agent $a$. This results in an amount of cooperating agents (friends) $\mathcal{F}_{a}$ of agent $a$ with:

$$
\begin{aligned}
\mathcal{F}_{a}= & \left\{b \in \mathcal{N}_{a} \mid \operatorname{div}(b, a) \leq q_{b}\right\} \\
\operatorname{div}(b, a)= & \left|u_{a}-u_{b}\right| \cdot g_{u, b}+\left|v_{a}-v_{b}\right| \cdot g_{v, b} \\
& +\left|w_{a}-w_{b}\right| \cdot g_{w, b}
\end{aligned}
$$

Heuristic 2 is implemented through the following procedure: all agents adapt their cultural traits to the traits of the best agent in their neighbourhood. With the probability $\rho_{a}$ agent $a$ adapts his traits to his neighbour $b^{*} \in \mathcal{N}_{a}$ with the highest score $s_{b}$, as fol- lowing:

$$
\begin{gathered}
u_{a}^{\prime}= \begin{cases}u_{a}+\left\lceil\left(u_{b}-u_{a}\right) \cdot\left(1-g_{u, a}\right)\right\rceil, & \text { iff } u_{a} \leq u_{b} \\
u_{a}+\left\lfloor\left(u_{b}-u_{a}\right) \cdot\left(1-g_{u, a}\right)\right\rfloor, & \text { iff } u_{a}>u_{b}\end{cases} \\
v_{a}^{\prime}= \begin{cases}v_{a}+\left\lceil\left(v_{b}-v_{a}\right) \cdot\left(1-g_{v, a}\right)\right\rceil, & \text { iff } v_{a} \leq v_{b} \\
v_{a}+\left\lfloor\left(v_{b}-v_{a}\right) \cdot\left(1-g_{v, a}\right)\right\rfloor, & \text { iff } v_{a}>v_{b}\end{cases} \\
w_{a}^{\prime}= \begin{cases}w_{a}+\left\lceil\left(w_{b}-w_{a}\right) \cdot\left(1-g_{w, a}\right)\right\rceil, & \text { iff } w_{a} \leq w_{b} \\
w_{a}+\left\lfloor\left(w_{b}-w_{a}\right) \cdot\left(1-g_{w, a}\right)\right\rfloor, & \text { iff } w_{a}>w_{b}\end{cases}
\end{gathered}
$$

therefore $\rho_{a}$ is defined by:

$$
\rho_{a}=P(\text { Adapt. })= \begin{cases}\left(1-\frac{1}{1+\left(s_{b}-s_{a}\right)}\right), & \text { iff } s_{b}>s_{a} \\ 0, & \text { else }\end{cases}
$$

To have the agents adapt their traits exclusively to the actual situation, the adaptations take place sorted in ascending order according to the scores (see Obervation 1).

\section{Observation 1 (X-Over-Freedom of Adaptations)}

If an agent a adapts his cultural traits to the traits of agent $b$ then there exists no agent $c$ who still would need to adapt to the traits of agent a. If there exists such an agent $c$, then as a necessary condition, agent $a$ is more successful than agent $c$. Furthermore, the score of agent $a$ is greater than the score of agent $s$, i.e. $s_{c}<s_{a}$. As $\mathcal{A}$ is sorted ascendingly according to $s$, agent $c$ is dealt with before agent $a$.

\subsection{Scenario Description}

In every round, all agents evaluate their neighbourhood and in particular determine the amount of neighbours which are willing to cooperate (friends $\mathcal{F}$ ). Subsequently all agents get $(|\mathcal{N}|+1)$ jobs to solve. An agent has no limit with regard to the amount of jobs he is able to solve per round. Only this agent to whom the job was assigned is rewarded, while the others, who were requested and accepted to help, have to pay the costs. However, if an agent is not able to find enough cooperating partners to solve a job, the job will be discarded. At the end of each round every agent identifies his best neighbour (role model) and possibly adapts his cultural traits. For the outline see Algorithm 1. 


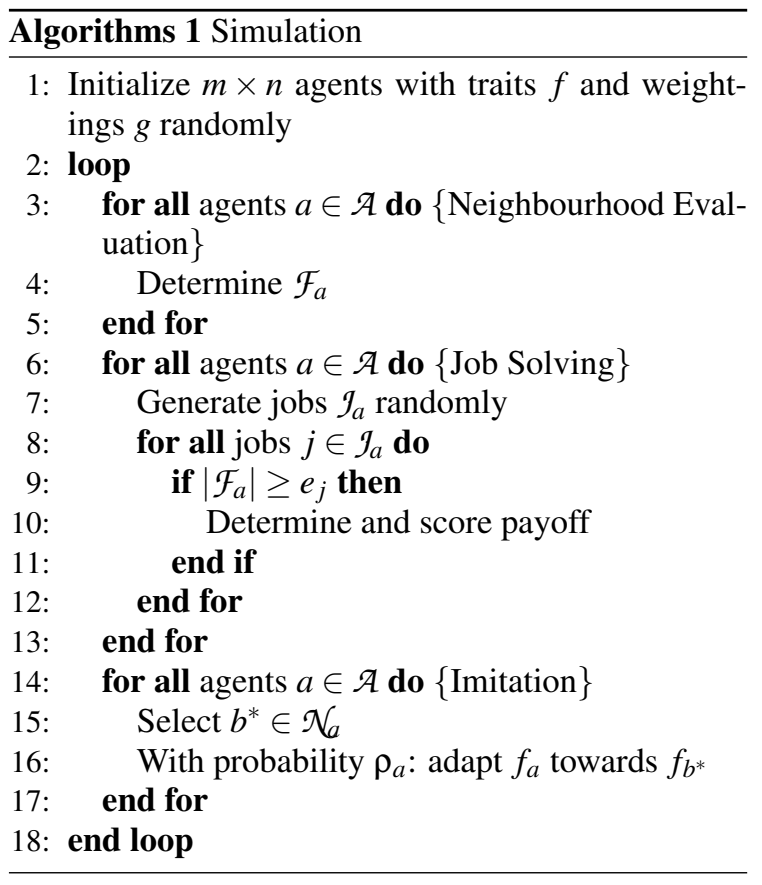

\section{ANALYTICAL APPROACH}

In this section the propagation of cultural traits will be analysed in detail. Klemm et al. have empirically investigated that a propagation of cultural traits in a multiagent system can lead to a globalisation (all agents become increasingly homogeneous) or a polarisation (many small clusters) (Klemm et al., 2005). By the MAS introduced in section 2, we analyse the propagation and thereby examine small cases of the adaptation convergence in detail. Subsequently, we will explore how many adaptation steps need to be performed until the cultural traits become adequately similar. For a universal analysis the criterion adequately similar is set on identical - with a deviation of 0 . Moreover, due to a restricted definition, only the agents which are neighbours - thus know each other and are able to interact - are considered. In the smallest case (by disregarding the neighbourhood relations) only two agents will interact with and imitate each other.

\subsection{Convergency in 1 to 1 Adaptation}

Taken into consideration are two agents $a \in \mathcal{A}$ and $b \in \mathcal{A}$ with the assumption that $a$ and $b$ only adapt to each other in each round. Accordingly, three cases can be differentiated (see Figure 2):

a. Agent $a$ adapts his cultural traits only to the traits of agent $b$. b. Agent $a$ and $b$ mutually adapt their cultural traits.

c. Agent $b$ only adapts his cultural traits to the traits of agent $a$.

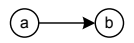

(a) Agent $a$ to $b$

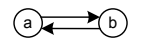

(b) Mutually

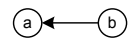

(c) Agent $b$ to $a$
Figure 2: Cases of the 1 to 1 adaptation.

Subsequently, the analysis is restricted to the cultural trait $u$. The analysis of the cultural traits $v$ and $w$ follows analogously. As additional requirement applies: $g_{u, a} \geq g_{u, b}$. According to Section 2.2 the distance $\delta=\left|u_{b}-u_{a}\right|$ can be defined and the adaptation formula can be simplified (for a better readability $\gamma=g_{u, a}$ is defined). According to case (a) agent $a$ exclusively adapts to agent $b$, therefore it follows (see Observation 2):

$$
\delta_{t+1}=\delta_{t}+\left\lfloor\left(0-\delta_{t}\right) \cdot(1-\gamma)\right\rfloor=\left\lfloor\delta_{t} \gamma\right\rfloor
$$

Observation 2 For $u_{b}$ and $u_{a}$ applies: $u \in\{1, \ldots, z\}$. The adaptation is performed in the direction of $u_{b}$, therefore in this adaptation step $u_{a}$ and therewith $\delta$ do change but $u_{b}$ remains constant.

From $\delta=\left|u_{b}-u_{a}\right|$ and $u_{a} \leq u_{b}$ follows: $\delta=u_{b}-u_{a}$ and $-\delta+u_{b}=u_{a}$. Consequently applies:

$$
\begin{aligned}
u_{a_{t+1}}= & u_{a_{t}}+\left\lceil\left(u_{b}-u_{a_{t}}\right) \cdot\left(1-g_{u, a}\right)\right\rceil \\
-\delta_{t+1}+u_{b}= & -\delta_{t}+u_{b} \\
& +\left\lceil\left(u_{b}-\left(-\delta_{t}+u_{b}\right)\right) \cdot\left(1-g_{u, a}\right)\right\rceil \\
-\delta_{t+1}+u_{b}= & -\delta_{t}+u_{b} \\
& +\left\lceil\left(u_{b}+\delta_{t}-u_{b}\right) \cdot\left(1-g_{u, a}\right)\right\rceil \\
-\delta_{t+1}= & -\delta_{t}+\left\lceil\left(0+\delta_{t}\right) \cdot\left(1-g_{u, a}\right)\right\rceil \\
\delta_{t+1}= & \delta_{t}+\left\lfloor\left(-\left(0+\delta_{t}\right) \cdot\left(1-g_{u, a}\right)\right)\right\rfloor \\
\delta_{t+1}= & \delta_{t}+\left\lfloor\left(0-\delta_{t}\right) \cdot\left(1-g_{u, a}\right)\right\rfloor
\end{aligned}
$$

From $\delta=\left|u_{b}-u_{a}\right|$ and $u_{a}>u_{b}$ follows: $\delta=u_{a}-u_{b}$ and $\delta+u_{b}=u_{a}$. Consequently applies:

$$
\begin{aligned}
u_{a_{t+1}} & =u_{a_{t}}+\left\lfloor\left(u_{b}-u_{a_{t}}\right) \cdot\left(1-g_{u, a}\right)\right\rfloor \\
\delta_{t+1}+u_{b} & =\delta_{t}+u_{b}+\left\lfloor\left(u_{b}-\left(\delta_{t}+u_{b}\right)\right) \cdot\left(1-g_{u, a}\right)\right\rfloor \\
\delta_{t+1}+u_{b} & =\delta_{t}+u_{b}+\left\lfloor\left(u_{b}-\delta_{t}-u_{b}\right) \cdot\left(1-g_{u, a}\right)\right\rfloor \\
\delta_{t+1} & =\delta_{t}+\left\lfloor\left(0-\delta_{t}\right) \cdot\left(1-g_{u, a}\right)\right\rfloor
\end{aligned}
$$

With the simplification $\gamma=g_{u, a}$ it follows:

$$
\delta_{t+1}=\delta_{t}+\left\lfloor\left(0-\delta_{t}\right) \cdot(1-\gamma)\right\rfloor
$$

Every adaptation step is decreasing the distance $\delta$ depending on the weighting $\gamma$ of the adapting agent. Keeping in mind that $\gamma \in] 0,1[$, the adaptation steps 
result in:

$$
\begin{aligned}
& \text { step } 1:\lfloor\delta \gamma\rfloor \\
& \text { step 2: }\lfloor\delta \gamma\rfloor \cdot \gamma\rfloor \leq\left\lfloor\delta \gamma^{2}\right\rfloor \\
& \text { step } 3:\left\lfloor\left\lfloor\delta \gamma^{2}\right\rfloor \cdot \gamma\right\rfloor \leq\left\lfloor\delta \gamma^{3}\right\rfloor \\
& \ldots \\
& \text { step } t:\left\lfloor\delta \gamma^{\dagger}\right\rfloor
\end{aligned}
$$

In the next step the point of time in which the cultural traits $(u)$ of the agent $a$ and $b$ become equal is searched for. That is the number $\tau$ of the adaptation steps according to which the difference is $\delta=0$. A sufficient condition is:

$$
\begin{aligned}
&\left\lfloor\delta \gamma^{\tau}\right\rfloor=0 \\
& \delta \gamma^{\tau}<1 \\
& \delta<\frac{1}{\gamma^{\tau}}=\left(\gamma^{-\tau}\right) \\
& \log _{\left(\gamma^{-1}\right)}(\delta)=\frac{\lg (\delta)}{\lg \left(\gamma^{-1}\right)}=\frac{\lg (\delta)}{-\lg (\gamma)}<\tau \\
& {\left[\frac{\lg (\delta)}{-\lg (\gamma)}\right]=\tau }
\end{aligned}
$$

The derivation for case (c) follows analogously. The case (b) can be estimated with case (a), as the adaptation cannot take longer at the same distance $\delta$ and a smaller or equal weighting (see Observation 3).

\section{Observation 3 (Duration of 1 to 1 Adaptation)}

With the condition $g_{u, a} \geq g_{u, b}$, it follows: The adaptation of the cultural traits of agent a to the traits of agents $b$ takes at least as long as a mutual adaptation or the adaptation of the cultural traits of agent $b$ to the traits of agent $a$. Let us assume, at any point $t^{\prime}$ of the adaptation, agent $b$ adapts his cultural traits to the traits of agent $a$. However, as agent $b$ has a weighting of $g_{u, b} \leq g_{u, a}$ at his command it follows directly that $\delta_{t^{\prime}+1}=\delta_{t^{\prime}}+\left\lfloor\left(0-\delta_{t^{\prime}}\right) \cdot\left(1-g_{u, b}\right)\right.$ leads to an approximation that is larger than or equal to the approximation of the inverted case a to $b$. This observation implies that at any point of time an adaptation from $b$ to a does not cannot need more steps.

Thus, case (a) is the worst case in the 1 to 1 convergence. If the weightings of the cultural traits are

\begin{tabular}{|c|c|c|c|c|c|c|c|}
\hline $\mathrm{t}$ & $u_{b_{i}}$ & $u_{a}$ & $u_{b_{j}}$ & $\mathrm{t}$ & $u_{b_{i}}$ & $u_{a}$ & $u_{b_{j}}$ \\
\hline 0 & 1 & 5 & 9 & 0 & 1 & 5 & 1 \\
\hline 1 & 1 & 2 & 6 & 1 & 1 & 2 & 4 \\
\hline 2 & 1 & 1 & 3 & 2 & 1 & 1 & 3 \\
\hline 3 & 1 & 1 & 2 & 3 & 1 & 1 & 2 \\
\hline 4 & 1 & 1 & 1 & 4 & 1 & 1 & 1 \\
\hline
\end{tabular}
equal, then the worst case is equivalent to the best case: every agent reduces the distance with about the same ratio.

\subsection{Convergence in $k$ to 1 Adaptation}

If those $k$ agents are considered, which interact with agent $a$, three cases of convergence can be distinguished as well (see Figure 3). The number $k$ of
Table 1: Examples for case (a) of the $k$ to 1 adaptation.

agents in consideration depends on the neighbourhood relation, which implies that some agents in the world can interact with agent $a$.

a. Agent $a$ adapts his cultural traits only to the traits of agent $b_{i}$ with $i \in\{1, \ldots, k\}$, while all other agents $b_{j}$ with $j \in\{1, \ldots, i-1, i+1, k\}$ adapt their traits to the traits of agent $a$.

b. The agents $a$ and $b_{i}$ with $i \in\{1, \ldots, k\}$ mutually adapt their cultural traits to each other. All other agents adapt their traits to the traits of agent $a$.

c. All agents $b_{i}$ with $i \in\{1, \ldots, k\}$ adapt their cultural traits only to the traits of agent $a$.

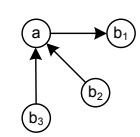

(a) Agent $a$ to $b_{i}$

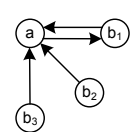

(b) Mutually

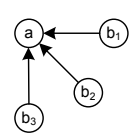

(c) Agent $b_{i}$ to $a$
Figure 3: Cases of the $k$ to 1 adaptation.

For the analysis of case (c) the equation of the 1 to 1 adaptation for all agents $\left(b_{1}, \ldots, b_{k}\right)$ can be adopted. However, the number of adaptation steps $\tau$ until convergence depends on the largest distance of all agents towards agent $a$.

$$
\begin{aligned}
& \tau=\max \left(\tau\left(b_{1}\right), \ldots, \tau\left(b_{k}\right)\right), \\
& \left\lceil\frac{\lg \left(\delta_{i}\right)}{-\lg \left(\gamma_{i}\right)}\right\rceil=\tau\left(b_{i}\right), \quad \delta_{i}=\left|u_{a}-u_{b_{i}}\right|, \quad \gamma_{i}=g_{u, b_{i}}
\end{aligned}
$$

In the cases (a) and (b) it needs to be considered that the cultural trait value (of $u$ ) of the agent $b_{i}$ can be smaller than the cultural trait value of agent $a$ and his cultural trait value can be smaller than the values of another agent $b_{j}$ (see Table1, left side). Another possible extreme is that the agents $b_{i}$ and $b_{j}$ have identical cultural trait values and do equally differ from agent $a$ (see example in Table 1, right side). Subsequently, the adaptation of $b_{j}$ over $a$ to $b_{i}$ needs to be computed. For that reason the number of adaptation steps can be 
added up and descriped as following:

$$
\begin{aligned}
& \tau=\tau\left(b_{i}\right) \\
& \quad+\max \left(\tau\left(b_{1}\right), \ldots, \tau\left(b_{i-1}\right), \tau\left(b_{i+1}\right), \ldots, \tau\left(b_{k}\right)\right) \\
& \left\lceil\frac{\lg \left(\delta_{i}\right)}{-\lg \left(\gamma_{i}\right)}\right\rceil=t\left(b_{i}\right), \quad \delta_{i}=\left|u_{a}-u_{b_{i}}\right|, \quad \gamma_{i}=g_{u, b_{i}}
\end{aligned}
$$

\subsection{Convergence in Unrestricted Adaptation}

To expand the observation of the convergence in unrestricted adaptation of finite $k$ agents, it is important to emphasise that role models can change in the course of the adaptation or even after a complete adaptation to a particular agent. Due to this circumstance two cases, which need to be analysed separately, emerge (see Figure 4).

a. For all agents applies: The role models do not change, although it is possible that several agents share the same model.

b. The role models of one agent can change. It is possible, in particular, that the models alternate cyclically.

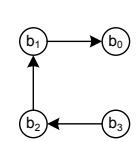

(a) Identical order

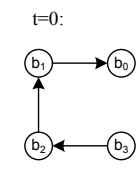

(b) Alternating order
Figure 4: Cases of the unrestricted adaptation.

To be able to analyse an unrestricted adaptation between $k$ agents with identical order (case (a)), a decomposition of the problem can be used. Due to the cyclic freedom of a model-follower relation (see Observation 1 ) for every model a $k$ to 1 convergence to his followers can be computated. As all adaptations to different role models happen simultaneously, an upper bound can be identified:

$\tau=2 \cdot \max \left(\tau\left(b_{1}\right), \ldots, \tau\left(b_{i-1}\right), \tau\left(b_{i+1}\right), \ldots, \tau\left(b_{k}\right)\right)-1$

$$
\left\lceil\frac{\lg \left(\delta_{i}\right)}{-\lg \left(\gamma_{i}\right)}\right\rceil=\tau\left(b_{i}\right), \quad \delta_{i}=\left|u_{a}-u_{b_{i}}\right|, \quad \gamma_{i}=g_{u, b_{i}}
$$

An alternating adaptation between $k$ agents (case (b)) does not result in a guaranteed bound for a convergence. In the worst case the order of the models can vary after each round, so that the same cultural trait values remain in various agents. Table 2 shows an example for such an arrangement which could never lead to a convergence.
Table 2: Example for case (b) of the unrestricted adaptation.

\begin{tabular}{ccccccccc}
\hline \multirow{2}{*}{} & \multicolumn{3}{c}{ Agent $b_{0}$} & \multicolumn{3}{c}{ Agent $b_{1}$} & \multicolumn{3}{c}{ Agent $b_{2}$} & \multicolumn{2}{c}{ Agent $b_{3}$} \\
& $u$ & RM & $u$ & RM & $u$ & RM & $u$ & RM \\
\hline 0 & 1 & - & 4 & $b_{0}$ & 3 & $b_{1}$ & 2 & $b_{2}$ \\
1 & 1 & - & 2 & $b_{3}$ & 4 & $b_{0}$ & 3 & $b_{2}$ \\
2 & 1 & - & 3 & $b_{3}$ & 2 & $b_{1}$ & 4 & $b_{0}$ \\
3 & 1 & - & 4 & $b_{0}$ & 3 & $b_{1}$ & 2 & $b_{2}$ \\
\hline \multicolumn{3}{c}{$g_{u, b_{i}}=\frac{1}{3}, \forall b_{i} \in\left\{b_{0}, \ldots, b_{3}\right\}, \mathrm{RM}=$ Role Model }
\end{tabular}

As a result it can be stated that a convergence to identical cultural traits can emerge, if a single agent is the role model during the adaptation steps or if there exists an invariant hierarchy between the agents. If there are several models which also alternate, then a convergence cannot be guaranteed.

\section{EMPIRICAL VALIDATION}

This section presents some empirical results to underline our analytical approach. We have simulated a lattice with $64 \cdot 64=4096$ agents over 500 rounds in a framework developed particularly for this purpose. The range dimension of cultural trait values was set to $z=64$ whereby the cultural trait values of all agents were situated in $\{1, \ldots, 64\}$. All cultural traits were weighted equally with $g_{u}=g_{v}=g_{w}=\frac{1}{3}$. According to section 2.3, the agents had to solve jobs and were able to adapt to their neighbours.

\subsection{Influence of Altruism Threshold}

Subsequently, we will describe the results of the variation of the altruism threshold $q$, which was the same for all agents. The graphs in Figure 5 show the first 100 rounds of the simulation and are the results of 100 independent runs, which were averaged.

For a configuration of the altruism threshold with $q=24$, almost $100 \%$ of the jobs were already solved after 25 rounds. In addition, in the case of such altruistic agents, a stable state is reached soon, so that after a few rounds a solved job rate of $97 \%$ is reached. Every smaller setting of $q$ leads to a slower development towards stable states and also to a smaller job solving rate. Nonetheless, the simulation shows that even for small values of $q$ a lower bound with $72 \%$ of solved jobs is reached: The solved job rate of $q=0$ and $q=4$ runs identically after 30 rounds - the only difference is a slower convergence to this stable state for $q=0$.

To measure the intensity of the cultural trait propagation, we determined and averaged the extent of 


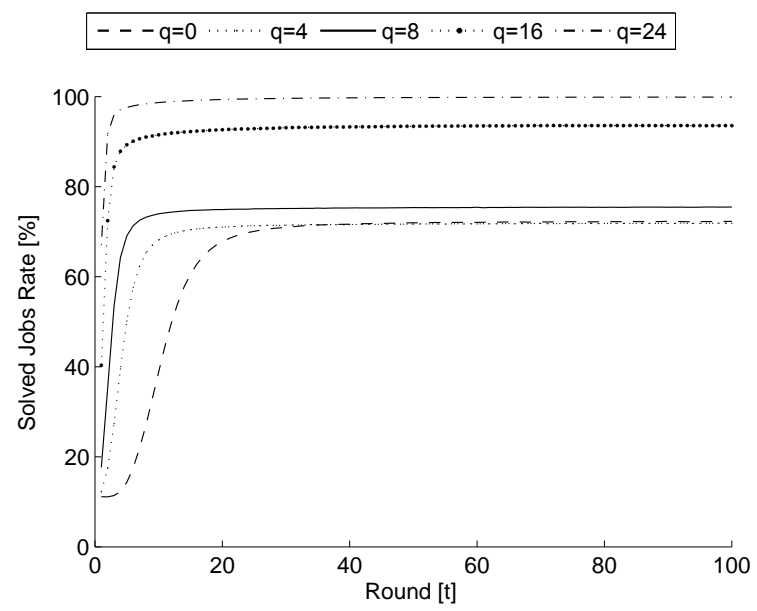

(a) Job solving: Jobs solved rate per round.

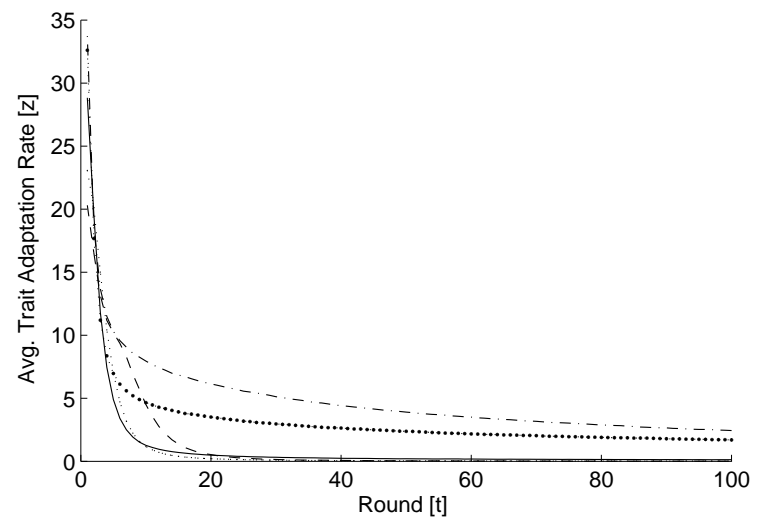

(b) Cultural trait propagation: Average trait adaptation rate per round.

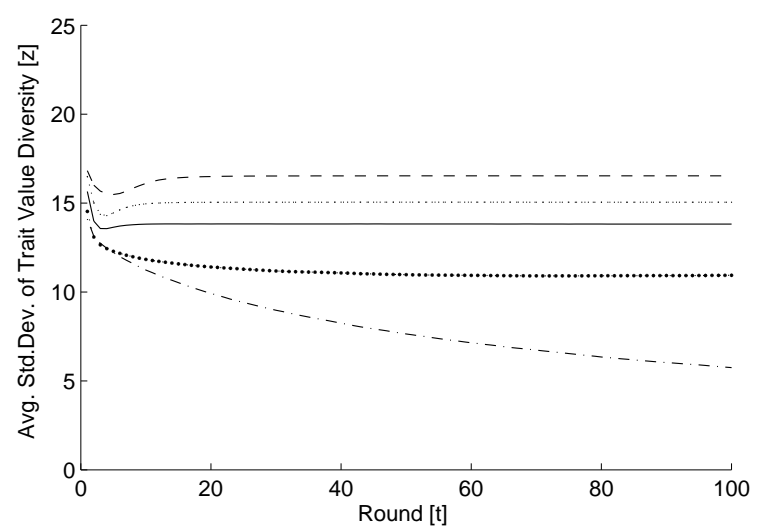

(b) Cultural trait propagation: Average standard deviation of cultural trait value diversity per round.

Figure 5: Simulation results for variation of the altruism threshold $q$. the adaptation steps for every trait and agent in every round. The cultural trait propagation in the settings $q=0, q=4$ and $q=8$ comes to a stagnancy: after 30 rounds no distinctive adaptations can be identified. In this process the curves approximate varyingly quick to the zero line: For $q=8$ most quickly and for $q=4$ and $q=0$ increasingly slower. For the settings $q=16$ and $q=24$ a continuous adaptation, which only slowly gets smaller, takes place.

For each cultural trait, we computed the mean and the standard deviation of the trait values over all agents to measure the 'trait diversity'. Averaged over the traits $u, v$ and $w$, the standard deviation of the cultural trait diversity points out that the trait variety in small settings of $q$ hardly decreases. After a minor adaptation wave in the first 10 rounds, the diversity starts to increase and finally remains consistent. For larger values of $q$ the cultural trait variety continuously decreases until it stagnates for $q=16$ at $\sigma=11.78$ and for $q=24$ at $\sigma=4.34$.

\subsection{Shape of Polarisation and Globalization}

In Figure 6 snapshots of the state of the world at the points of time $t=10$ and $t=100$ are compared (always for the upper and lower extreme of the varied parameter $q$ ). Those snapshots were recorded for typical runs of the parameter characteristics and are representative for all runs. Each cell represents an agent, for whom the cultural trait values were transferred to the RGB-colour-values $($ red $=256 / z \cdot(u-1)$, green $=$ $256 / z \cdot(v-1)$, blue $=256 / z \cdot(w-1))$. In the initial state $t=0$ snapshots show a unstructured distribution of all colours (not displayed). The purpose of this illustration is a visual comparison for surveying trait similarities in parts of and in the whole world respectively.

Those snapshots of the states of the world reveal that for small $q$ many small sections with similar cultural trait values emerge. Already after ten rounds a polarised world for $q=0$ has formed, which contains clusters that are almost as large as a Mooreneighbourhood ( $3 \times 3$ agents). For large $q$ the margins of smaller areas appear blurred, and with increasing numbers of rounds continuously larger areas with average cultural trait values emerge. In this process the cultural traits gradually spread over the whole world: the agents don't face a stable cultural trait configuration until the whole world has become homogeneous. Accordingly, a globalisation evolves. 


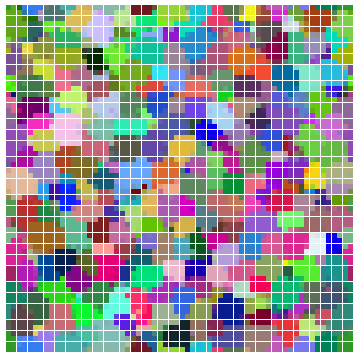

(a1) $q=0, t=10$

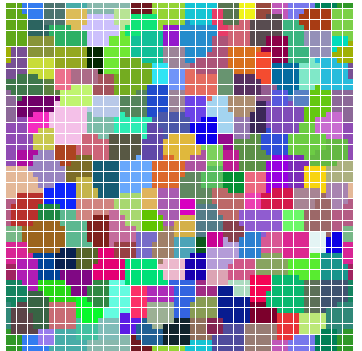

(a2) $q=0, t=100$

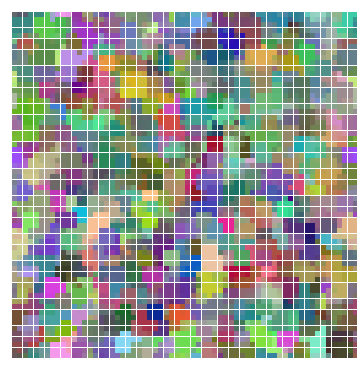

(b1) $q=24, t=10$

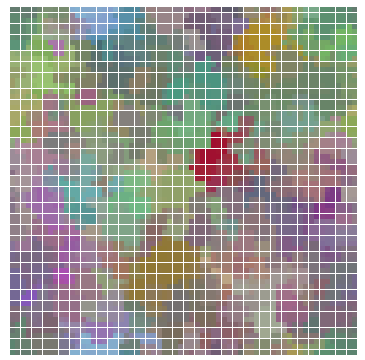

(b2) $q=24, t=100$
Figure 6: Comparison of state snapshots of typical runs for variation of the altruism threshold $q$.

\section{CONCLUSION \& FUTURE WORK}

To determine cooperation in multiagent systems with cultural traits, this work explored trait propagation and its interaction with cooperation. In contrast to only empirical work (Hales, 2001; Klemm et al., 2005), our analytical approach showed that in a MAS with cultural traits: 1 . It is possible that distinctive traits will spread completely over a population and converge to a specific traits setting. 2. Under certain conditions, a propagation can stagnate. Through empirical experiments we have found that the basic disposition for altruistic behaviour of course has a major influence on the propagation of traits and thus in second place positively affects the cooperation and vice versa. Above all, these results confirm the claim by (Klemm et al., 2005) that there is a threshold, which divides between polarisation and globalisation. If the willingness to cooperate, and thereby confidence and sympathy are generally high, the cultural traits spread quickly over the whole population and evoke more confidence and sympathy. In the case of very selfish agents who are unwilling to cooperate, the existence of more successful agents in the neighbourhood leads to a only local cultural trait propagation and thereby to more cooperation within this cluster.

In future work agents can to a minor degree randomly replace cultural traits through completely dif- ferent ones (mutation) and will be able to get to know new agents and abandon existing contacts (mobility). Furthermore, agents will possess various abilities, so that jobs, which require various abilities, can be solved only by distinctive cooperation partners (see (Eberling, 2009; Edmonds et al., 2009)). Additional work in this field could contribute to explain the accomplishment of cooperation in networks with many individuals and many different cultural traits.

\section{REFERENCES}

Allison, P. D. (1992). The Cultural Evolution of Beneficent Norms. Social Forces, 71(2):279-301.

Axelrod, R. (1986). An Evolutionary Approach to Norms. The American Political Science Review, 80(4):10951111.

Axelrod, R. (1997). The Complexity of Cooperation. Princeton University Press.

Axelrod, R. and Hamilton, W. D. (1981). The Evolution of Cooperation. Science, 211(4489):1390-1396.

Binmore, K. (1998). Game Theory and the Social Contract: Just Playing, volume 2. The MIT Press.

Boyd, R. and Richerson, P. J. (1985). Culture and the Evolutionary Process. University of Chicago Press.

Cialdini, R. B. (1994). Influence: The Psychology of Persuasion. New York: Quill.

Dawkins, R. (1976). The Selfish Gene. Oxford University Press.

Delgado, J. (2002). Emergence of Social Conventions in Complex Networks. Artificial Intelligence, 141:171185.

Eberling, M. (2009). Towards Determining Cooperation based on Multiple Criteria. In Mertsching, B., Hund, M., and Aziz, Z., editors, KI 2009. LNCS, vol. 5803, pages 548-555. Springer, Paderborn.

Edmonds, B., Norling, E., and Hales, D. (2009). Towards the Evolution of Social Structure. Computational \& Mathematical Organization Theory, 15(2):78-94.

Hales, D. (2001). Tag Based Co-operation in Artificial Societies. PhD thesis, Department of Computer Science, University of Essex, UK.

Heylighen, F. (1992). 'Selfish' Memes and the Evolution of Cooperation. Journal of Ideas, 2(4):70-76.

Klemm, K., Eguluza, V. M., Torala, R., and Miguela, M. S. (2005). Globalization, Polarization and Cultural Drift. J. Economic Dynamics and Control, 29:321-334.

Noble, J. and Franks, D. W. (2004). Social Learning in a Multi-Agent System. Computing and Informatics, 22(6):561-574.

Shoham, Y. and Leyton-Brown, K. (2008). Multiagent Systems: Algorithmic, Game Theoretic, and Logical Foundations. Cambridge University Press.

Trivers, R. L. (1971). The Evolution of Reciprocal Altruism. The Quarterly Review of Biology, 46(1):35-57. 